\title{
Antibody and immune memory persistence post infant hepatitis $B$ vaccination
}

\author{
This article was published in the following Dove Press journal: \\ Patient Preference and Adherence \\ 26 September 2013 \\ Number of times this article has been viewed
}

\section{Shuaibu A Hudu',2 \\ Yasmin A Malik ${ }^{3}$ \\ Mohd Taib Niazlin' \\ Nabil S Harmal',4 \\ Ariza Adnan ${ }^{5}$ \\ Ahmed S Alshrari' \\ Zamberi Sekawi'}

'Department of Medical Microbiology and Parasitology, Faculty of Medicine and Health Sciences, Universiti Putra Malaysia, Selangor, Malaysia; ${ }^{2}$ Department of Pathology and Medical Microbiology, College of Health Sciences, Usmanu Danfodiyo University Sokoto, Sokoto State, Nigeria; ${ }^{3}$ Department of Clinical Science, Faculty of Medicine and Health Sciences, Universiti Tunku Abdul Rahman, Selangor, Malaysia; ${ }^{4}$ Department of Medical Microbiology, Faculty of Medicine and Health Sciences, Sana'a University, Sana'a, Yemen; ${ }^{5}$ Cluster of Laboratory Medical Sciences, Faculty of Medicine Universiti Teknologi MARA, Sungai Buloh, Selangor, Malaysia
Correspondence: Zamberi Sekawi Department of Medical Microbiology and Parasitology, Faculty of Medicine and Health Sciences, Universiti Putra Malaysia 43400 UPM Serdang, Selangor Malaysia

Tel +6039847 2365

Fax +60389413802

Email zamberi@medic.upm.edu.my
Objectives: This study aimed to evaluate the level of hepatitis B immunity among undergraduate students 23 years after commencement of the nationwide hepatitis B childhood immunization program in Malaysia.

Methods: A total of 402 serum samples obtained from volunteer undergraduate students were screened for the presence of hepatitis B surface antibodies using qualitative ELISA.

Results: Results showed that $62.7 \%$ of volunteers had protective anti-hepatitis B surface antigens ( $\geq 10 \mathrm{IU} / \mathrm{L}$ ), of whom $67.9 \%$ received three doses of the vaccine. The estimated post-vaccination immunity was found to be at least 20 years, indicating persistent immunity against hepatitis $\mathrm{B}$ and a significant association $(P<0.05)$ with duration of vaccination. Anamnestic response 1 month post-hepatitis B booster was $94.0 \%$ and highly significant $(P<0.01)$. Isolated antihepatitis B core antigen (anti-HBc) prevalence was found to be $5.0 \%$, all having had a positive anamnestic response.

Conclusion: Immunity after primary vaccination with hepatitis B recombinant vaccine persists for at least 20 years post-vaccination, with significant association with the number of vaccinations. Furthermore, the presence of anamnestic response to booster vaccine indicates long-lasting immunity despite decreasing antibody levels; therefore, the need for hepatitis B vaccine boosters may not be of significant benefit after complete infant vaccination.

Keywords: hepatitis B vaccination, persistent immunity, anamnestic response, booster vaccination

\section{Introduction}

The hepatitis B surface antigen (HBsAg) contains a region that is common to all hepatitis B subtypes called the "a" determinant. ${ }^{1}$ The "a" determinant region (121-149 codons) is a two-loop structure located inside the major hydrophilic region of the surface protein, and serves as the key target for the neutralizing antibodies produced following vaccination or natural infection with the hepatitis B virus (HBV)., ${ }^{2,3}$ Antibodies against hepatitis B surface antigen (anti-HBs) provide protection against hepatitis B infection, and thus, vaccination with HBsAg provides adequate immunity against hepatitis B. However, the question still remains of how long the immunity lasts. To achieve hepatitis B protection, two types of vaccines have been used: (1) a plasmaderived vaccine developed by purifying HBsAg from hepatitis B carrier serum which has been used for many years, but not without major limitations, such as the availability of raw materials, cost, and in some cases breakthrough infections; and (2) the recombinant hepatitis B vaccine which was put into use in the late 1980s and derived from yeast-based recombinant technology. The recombinant vaccine contains the S-domain 
of HBsAg with 226 sequences, which is a small non-glycosylated surface protein, excluding the pre-S domain. It has been found to be efficient and safe, with little or no harmful side effects. ${ }^{4}$ The immunity acquired after vaccination consists of two major components, humoral and cell-mediated immunity. The humoral immune response is mediated by antibodies as a result of vaccination or exposure to the viral antigen, and it includes both primary and secondary responses. ${ }^{5}$

Dendritic and other antigen presenting cells, as well as B and T lymphocytes, are necessary for the formation of antigen-specific antibodies. ${ }^{6}$ Hepatitis B vaccines induce a primary immune response aimed at producing more effective and faster secondary responses upon natural exposure to HBV. A serum antibody titer of no less than $10 \mathrm{IU} / \mathrm{L}$ is required to provide protection against hepatitis B infection. ${ }^{7}$ In Malaysia, 2.4 million people are estimated to be hepatitis B carriers and they continue to be a potential source of infection (Malaysian Liver Foundation. Hepatitis B: Fact sheet for doctors. [Cited 2005 Jun 29]. Available from: URL: http://www.liver.org.my), with a prevalence of $5.24 \%$ reported in healthy volunteers. ${ }^{8}$ Therefore, national policy on infant immunization was adopted in the late 1980s, which corresponded with the introduction of the recombinant vaccine. ${ }^{9}$ The hepatitis B vaccination was integrated into the childhood immunization schedule starting in 1989, consisting of three doses at birth, 1 month, and 5 months; successful childhood vaccination depended largely on long-lasting immunity into adulthood. However, studies evaluating the success and limitations of the infant hepatitis B vaccination program in terms of post-vaccination antibody response since the program's introduction are lacking. Hence, the objective of this study was to assess the persistence of immunity after hepatitis B infant vaccination with recombinant hepatitis B in Malaysia.

\section{Materials and methods}

\section{Study population}

A total of 402 volunteers were recruited in this study. All participants were undergraduate student volunteers born on or after 1989 (the year in which the hepatitis B vaccine was incorporated into the Malaysian childhood vaccination program), excluding students who were vaccinated in the intervening year and those born before 1989. Ethical approval was obtained from the Faculty of Medicine and Health Sciences Ethical Committee, Universiti Putra Malaysia. Written and informed consent was also obtained from all participants.

\section{Sample collection}

Venous blood $(5 \mathrm{~mL})$ was taken from all volunteers into a vacutainer tube containing clot activator (Becton, Dickinson and Company, Franklin Lakes, NJ, USA) using aseptic techniques. Serum was separated from the blood by centrifuging at $4000 \mathrm{rpm}$ for 10 minutes at room temperature (RT), and was kept at $-80^{\circ} \mathrm{C}$ until further analysis. Structured, self-administered questionnaires recorded age, sex, race, and marital status. Vaccine history and factors associated with post-vaccination immunity, such as body mass index, family history of liver cancer, and cigarette smoking, were also recorded.

\section{Enzyme-linked immunosorbent assay for anti-HBs, $\mathrm{HbsAg}$, and anti-HBc}

Serum samples and test kits were equilibrated to RT; the antiHBs and HBsAg status of subjects was determined using a commercial enzyme-linked immunosorbent assay (ELISA) kit according to the manufacturer's instruction (DRG International, Inc., Springfield Township, NJ, USA). Washing was done using an immune washer (Model \# 1575; Bio-Rad Laboratories, Inc., Hercules, CA, USA) and absorbance measured at $450 \mathrm{~nm}$ using ELISA reader version 6.4 (TECAN Magellan, Austria). The presence or absence of protective anti-HBs antibody was read using the optical density (OD), as recommended by manufacturers. The ELISA kit has a sensitivity of $10 \mathrm{IU} / \mathrm{L}$ and $\mathrm{OD} \geq 0.105$. All volunteers with anti-HBs titers $\geq 10 \mathrm{IU} / \mathrm{L}$ were considered to be immune, while those with anti-HBs $<10$ IU/L were considered not to have persistent immunity.

Antibodies against the hepatitis B core antigen (anti-HBc) were also tested by commercial ELISA kit (DRG International, Inc.) according to manufacturer's guide, and the test was repeated to exclude false positive results. The presence or absence of anti-HBc was determined by comparing the absorbance value of the specimen to a cut-off value calculated from negative controls provided in the ELISA kit.

\section{Hepatitis $B$ vaccine booster dose}

A booster dose with recombinant hepatitis B vaccine of $20 \mu \mathrm{g}$ (Euvax B; Sanofi S.A., Paris, France) was administered via intramuscular injection to individuals with undetectable anti-HBs (<10 IU/L). Anti-HBs was checked 4 weeks after the booster, and individuals with anti-HBs titers $\geq 10 \mathrm{IU} / \mathrm{L}$ were defined as positive anamnestic response, while those lacking anamnestic response were defined as non-respondent and given complete vaccination.

\section{Statistical analysis}

The association between duration of vaccination and immunity were evaluated using Pearson's chi-squared test at 
a $P<0.05$. A McNemar test of related samples and protective immunity at various ages post-primary vaccination was evaluated using SPSS statistics software version 21.0 (IBM Corporation, Armonk, NY, USA).

\section{Results}

The study flow and scheme are illustrated in Figure 1. Among the 402 subjects, 296 (73.6\%) were female and $106(26.4 \%)$ male. Subject demographics were as follows: 267 (66.4\%) Malay; 112 (27.9\%) Chinese; 9 (2.2\%) Indian; and $14(3.5 \%)$ other races. The majority of the study population $(59.7 \%, 240)$ received three doses of the hepatitis B vaccine according to the Malaysian vaccination schedule, while $69(17.2 \%)$ received two doses and 93 (23.1\%) one dose (Table 1). Hepatitis B antibody titers of the study population revealed that $252(62.7 \%)$ individuals had protective anti-HBs ( $\geq 10 \mathrm{IU} / \mathrm{L})$, wherein $67.9 \%(171 / 252)$ received three doses, $18.6 \%(47 / 252)$ two doses, and $13.5 \%(34 / 252)$ one dose. Moreover, 37.3\% (150/402) had an anti-HBs titer $<10 \mathrm{IU} / \mathrm{L}$ indicating lack of protection against HBV, and $56.7 \%(85 / 150)$ received only one dose of the vaccine. Immunity persisted for at least 2 decades, with the majority of the study group having protective anti-HBs titers compared to other groups, with the number of those protected declining as time since vaccination increased.

Pearson's chi-squared test showed a significant association $(P<0.05)$ between persistence of immunity, duration since vaccination, and number of doses, while no significant association was found with body mass index, race, family history of liver cancer, hepatitis B infection, blood transfusion, and cigarette smoking. Anti-HBc results showed that $5 \%$ (20/402) seroconverted to anti-HBc, of which the majority (55\%) received 3 doses of the vaccine, $20 \%$ two doses, and $25 \%$ one dose. However, none of the subjects were HBsAg
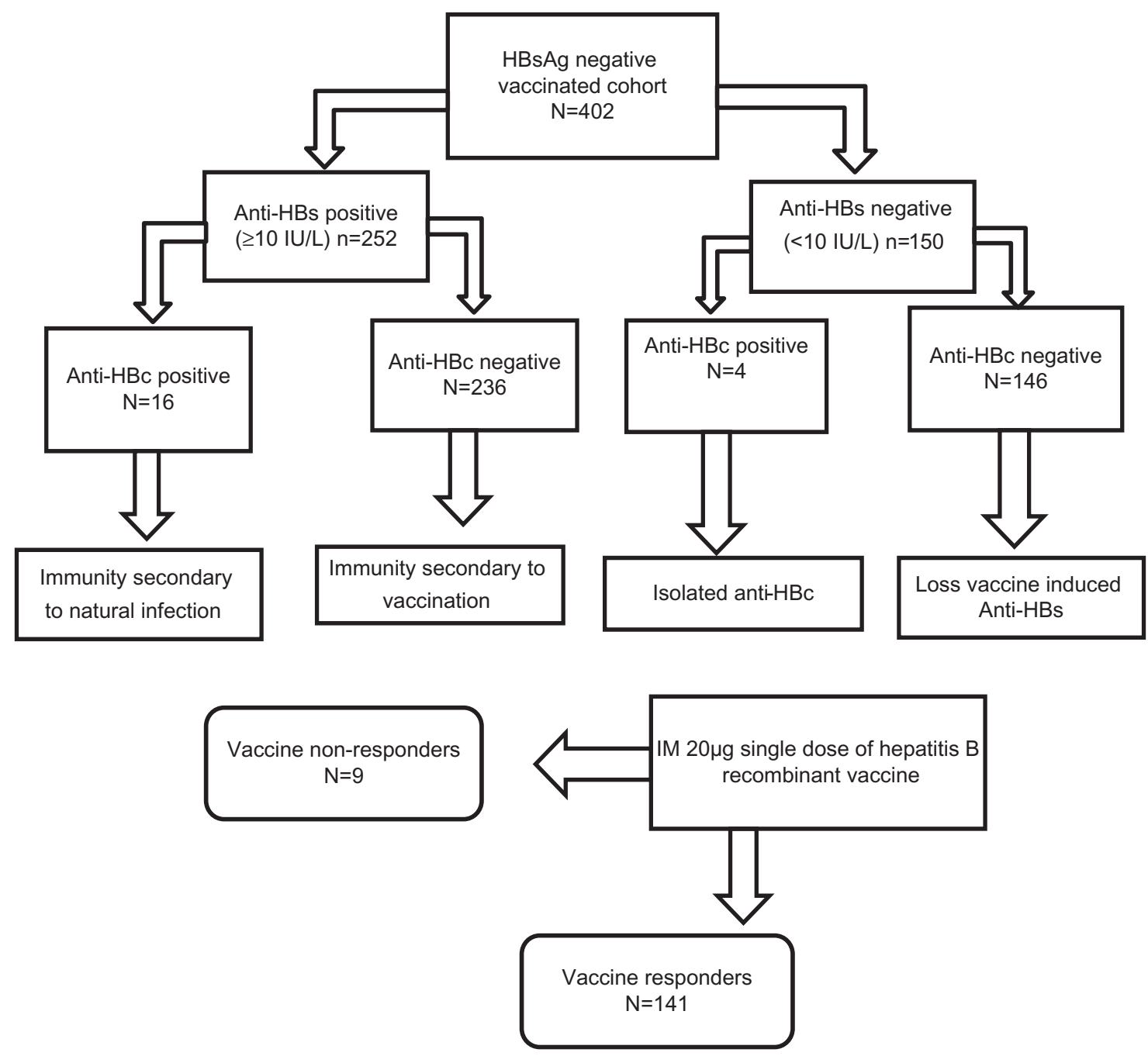

Figure I Schematic representation of study flow and outline.

Abbreviations: $\mathrm{HBsAg}$, hepatitis B surface antigens; anti-HBs, antibodies against hepatitis B surface antigen; anti-HBc, anti-hepatitis B core antigen; IM, intramuscular. 
Table I Hepatitis B antibody levels, number of vaccine doses, and core antigen in the vaccinated cohort with negative $\mathrm{HBsAg}$

\begin{tabular}{lllllll}
\hline $\begin{array}{l}\text { Hepatitis B } \\
\text { antibody titer }\end{array}$ & \multicolumn{3}{l}{$\begin{array}{l}\text { Hepatitis B core } \\
\text { antigen }\end{array}$} & & \multicolumn{3}{l}{$\begin{array}{l}\text { Number } \\
\text { of doses }\end{array}$} \\
\cline { 2 - 3 } \cline { 6 - 8 } & Positive & Negative & & I & $\mathbf{2}$ & $\mathbf{3}$ \\
\hline Positive $(\geq 10$ IU/L) & 16 & 236 & & 34 & 47 & 171 \\
Negative $(<10$ IU/L) & 4 & 146 & & 59 & 22 & 69 \\
Total & 20 & 382 & & 93 & 69 & 240 \\
\hline
\end{tabular}

Abbreviation: $\mathrm{HBsAg}$, hepatitis $B$ surface antigens.

positive (Table 1). Anamnestic response was positive in $94 \%$ (141/150), with only 6\% (9) non-respondent and McNemar test of related samples showing highly significant $(P<0.01)$ post-vaccination response to recombinant hepatitis $B$ vaccine (Sanofi S.A.).

\section{Discussion}

This study evaluates post-hepatitis B infant vaccination immunity 24 years following the introduction of the hepatitis B vaccination program in Malaysia. The level of anti-HBs required to provide effective protection against HBV after childhood vaccination with recombinant hepatitis $\mathrm{B}$ vaccine has been shown to be $10 \mathrm{IU} / \mathrm{L}$ in a previous controlled clinical trial. ${ }^{10}$ A study conducted in Thailand found that $83.9 \%$ of the vaccinated cohort had protective anti-HBs 20 years after infant vaccination, ${ }^{11}$ which is consistent with our findings and concurs with a number of studies that confirmed the persistence of antibodies (21-22 years) following infant vaccination with recombinant hepatitis B vaccine. ${ }^{12,13} \mathrm{~A}$ recent study in the UK showed that $84.6 \%$ of children immunized with three doses of the vaccine at infancy have persistent immunity, ${ }^{14}$ while our study found that $67.9 \%$ (171/252) of subjects who received three doses had persisting protective anti-HBs, with significant association to the number of doses $(P<0.05)$. Our results also revealed declining anti-HBs titers within a year of vaccination, similar to findings in previous related studies ${ }^{15-17}$ and in concurrence with findings in Brazil, which reported a positive correlation between anti-HBs titers after primary vaccination with the duration of the vaccination. ${ }^{18}$ Persistence of antibody has long been considered a marker for vaccine protection; nevertheless, a better understanding of immunology has led to the understanding that long-term protection is also conferred by immune memory cells. Several studies have examined humoral and cellular response to vaccine challenge, this study included.

Our results demonstrated that anamnestic response was positive in $94 \%(141 / 150)$ of study subjects; only
$6 \%$ (9) were non-responders, which is similar to the results in previous studies in Iran $(95.75 \%),{ }^{19}$ Alaska (79\%) ${ }^{20}$ Thailand (98.6\%), ${ }^{11}$ Taiwan $(95.9 \%),{ }^{21}$ and the UK (95.6\%). ${ }^{14}$ Several other studies have reported anamnestic response ranging from $60 \%$ to $75 \% .{ }^{22-24}$ In the present study, we recorded a $6 \%(9 / 150)$ non-responder rate, which is lower than the range reported in several other studies $(8 \%-21 \%) .{ }^{25-27}$ Thus, the presence of immune memory postinfant vaccination has been confirmed in this study, as well as other similar studies with anamnestic response, indicating persistence of memory beyond the duration of circulating antibodies. ${ }^{28}$ Malaysia was categorized as an intermediate endemic area, but due to the introduction of vaccination with effective coverage, they are now considered as a low endemic area; ${ }^{9}$ therefore, it has low a chance of natural boosters. However, the chances of natural boosters in this study population cannot be ruled out.

The rate of anti-HBc-positive subjects among the vaccinated cohort was found to be $5 \%(20 / 402)$, which is lower than a previous Italian report $(18.3 \%) ;{ }^{29}$ this finding may be due to differences in vaccine strategies. Moreover, in a similar study in the People's Republic of China, the rate of anti-HBc positivity was found to be lower in vaccinated compared to non-vaccinated subjects, ${ }^{30}$ but none of the sample was found to be HBsAg positive, which was similar to findings in Gambians. ${ }^{31}$ Current results demonstrated that $80 \%(16 / 20)$ of anti-HBc positive subjects were also positive for anti$\mathrm{HBs}$, indicating immunity through natural infection; similar to a study in Taiwan, ${ }^{32} 20 \%(4 / 20)$ were anti-HBc positive but anti-HBs negative (referred to as isolated anti-HBc, see Figure 1). Isolated anti-HBc may be a result of the following: (1) resolved natural infection with waning anti-HBs, referred to as 'type I isolated anti-HBc'; (2) false-positive anti-HBc, referred to as 'type II isolated anti-HBc'; (3) occult hepatitis B infection with undetectable anti-HBs, referred to as 'type III isolated anti-HBc'; or (4) resolved HBV antigenemia with undeveloped anti-HBs (window period), referred to as 'type IV isolated anti-HBc'. Anti-HBc may be due to cross-reacting non-specific antibodies. ${ }^{33,34}$

There were several limitations in this study. First, the lack of association with blood transfusion or smoking or body mass index might be due to low power of the study to detect such association. Secondly, data collected on the number of doses of vaccine received at infant was based on the parent information. Third, only qualitative ELISA test was used in this study; therefore, we were unable to measure anti-HBs seropositivity, measuring only seroprotection rates. Lastly, 
subjects with anti-HBs negatives and anti-HBc positives were not assessed for HBsAg after the booster dose.

\section{Conclusion}

Persistence of immunity after primary vaccination with hepatitis $\mathrm{B}$ recombinant vaccine persists for at least 20 years post-vaccination, with higher protection in those who received three doses of the vaccine compared to one or two doses. Anamnestic response was positive in $94 \%$, while an isolated anti-HBc rate of 5\% was found with no breakthrough infection. In view of the evidence presented in this study, the presence of anamnestic response to booster vaccine indicates long lasting immunity despite decreasing antibody levels; therefore the use hepatitis B vaccine boosters may not be of significant benefit after complete infant vaccination. Our data are essential for the evaluation of vaccination programs and strategies, forming a baseline for further studies. Hence, there is need for a large scale study involving children, adolescent, and adult populations in Malaysia.

\section{Acknowledgment}

This study was supported by Sanofi-Aventis Malaysia, in conjunction with the Faculty of Medicine and Health Sciences, Universiti Putra Malaysia.

\section{Disclosure}

The authors report no conflicts of interest in this work.

\section{References}

1. Howard CR, Allison LM. Hepatitis B surface antigen variation and protective immunity. Intervirology. 1995;38(1-2):35-40.

2. Waters JA, O'Rourke SM, Richardson SC, Papaevangelou G, Thomas HC. Qualitative analysis of the humoral immune response to the "a" determinant of HBs antigen after inoculation with plasma-derived or recombinant vaccine. J Med Virol. 1987;21(2):155-160.

3. Tong S, Kim KH, Chante C, Wands J, Li J. Hepatitis B Virus e Antigen Variants. Int J Med Sci. 2005;2(1):2-7.

4. Halliday ML, Rankin JG, Bristow NJ, Coates RA, Corey PJ, Strickler AC. A randomized double-blind clinical trial of a mammalian cell-derived recombinant DNA hepatitis B vaccine compared with a plasma-derived vaccine. Arch Intern Med. 1990;150(6):1195-1200.

5. Banatvala J, Van Damme P, Oehen S. Lifelong protection against hepatitis B: the role of vaccine immunogenicity in immune memory. Vaccine. 2000;19(7-8):877-885.

6. van Montfoort N, Mangsbo SM, Camps MG, et al. Circulating specific antibodies enhance systemic cross-priming by delivery of complexed antigen to dendritic cells in vivo. Eur J Immunol. 2012;42(3): 598-606.

7. Honorati MC, Facchini A. Immune response against HBsAg vaccine. World J Gastroenterol. 1998;4(6):464-466.

8. Merican I, Guan R, Amarapuka D, et al. Chronic hepatitis B virus infection in Asian countries. J Gastroenterol Hepatol. 2000;15(12): $1356-1361$.
9. Ng KP, Saw TL, Baki A, Rozainah K, Pang KW, Ramanathan M. Impact of the Expanded Program of Immunization against hepatitis B infection in school children in Malaysia. Med Microbiol Immunol. 2005;194(3): $163-168$.

10. Szmuness W, Stevens CE, Zang EA, Harley EJ, Kellner A. A controlled clinical trial of the efficacy of the hepatitis B vaccine (Heptavax B): a final report. Hepatology. 1981;1(5):377-385.

11. Poovorawan Y, Chongsrisawat V, Theamboonlers A, Leroux-Roels G, Crasta PD, Hardt K. Persistence and immune memory to hepatitis B vaccine 20 years after primary vaccination of Thai infants, born to $\mathrm{HBsAg}$ and HBeAg positive mothers. Hum Vaccin Immunother. 2012;8(7): 896-904.

12. Hammitt LL, Hennessy TW, Fiore AE, et al. Hepatitis B immunity in children vaccinated with recombinant hepatitis $B$ vaccine beginning at birth: a follow-up study at 15 years. Vaccine. 2007;25(39-40): 6958-6964.

13. But DY, Lai CL, Lim WL, Fung J, Wong DK, Yuen MF. Twenty-two years follow-up of a prospective randomized trial of hepatitis B vaccines without booster dose in children: Final report. Vaccine. 2008;26(51): 6587-6591.

14. Yates TA, Paranthaman K, Yu LM, et al. UK vaccination schedule: persistence of immunity to hepatitis B in children vaccinated after perinatal exposure. Arch Dis Child. 2013;98(6):429-433.

15. T, Fiore AE, Negus S, et al. Differences in response to a hepatitis B vaccine booster dose among Alaskan children and adolescents vaccinated during infancy. Pediatrics. 2007;120(2):e373-e381.

16. Bialek SR, Bower WA, Novak R, et al. Persistence of protection against hepatitis $\mathrm{B}$ virus infection among adolescents vaccinated with recombinant hepatitis $B$ vaccine beginning at birth: a 15-year follow-up study. Pediatr Infect Dis J. 2008;27(10):881-885.

17. Lao TT, Sahota DS, Suen SS, Chan PK, Leung TY. Impact of neonatal hepatitis $\mathrm{B}$ vaccination programme on age-specific prevalence of hepatitis B infection in teenage mothers in Hong Kong. Epidemiol Infect. 2012:1-9.

18. Alexandre KV, Martins RM, Souza MM, Rodrigues IM, Teles SA. Brazilian hepatitis B vaccine: a six-year follow-up in adolescents. Mem Inst Oswaldo Cruz. 2012;107(8):1060-1063.

19. Jafarzadeh A, Montazerifar SJ. Persistence of anti-HBs antibody and immunological memory in children vaccinated with hepatitis B vaccine at birth. J Ayub Med Coll Abbottabad. 2006;18(4):4-9.

20. Samandari T, Fiore AE, Negus S, et al. Differences in response to a hepatitis B vaccine booster dose among Alaskan children and adolescents vaccinated during infancy. Pediatrics. 2007;120(2):e373-e381.

21. Lu CY, Chiang BL, Chi WK, et al. Waning immunity to plasma-derived hepatitis $\mathrm{B}$ vaccine and the need for boosters 15 years after neonatal vaccination. Hepatology. 2004;40(6):1415-1420.

22. Chaves SS, Groeger J, Helgenberger L, et al. Improved anamnestic response among adolescents boosted with a higher dose of the hepatitis B vaccine. Vaccine. 2010;28(16):2860-2864

23. Leuridan E, Van Damme P. Hepatitis B and the need for a booster dose Clin Infect Dis. 2011;53(1):68-75.

24. Chaves SS, Fischer G, Groeger J, et al. Persistence of long-term immunity to hepatitis B among adolescents immunized at birth. Vaccine. 2012;30(9):1644-1649.

25. Ahad MA, Alim MA, Guho A, Islam QT, Azad KAK. Role of Booster Dose on Antibody Titer after Recombinant Hepatitis B Vaccination. Journal of Medicine. 2009;10(2):67-76.

26. Barash C, Conn MI, DiMarino AJ, Marzano J, Allen ML. Serologic hepatitis B immunity in vaccinated health care workers. Arch Intern Med. 1999;159(13):1481-1483.

27. Zeeshan M, Jabeen K, Ali AN, et al. Evaluation of immune response to Hepatitis B vaccine in health care workers at a tertiary care hospital in Pakistan: an observational prospective study. BMC Infect Dis. 2007;7:120.

28. No author listed. Are booster immunisations needed for lifelong hepatitis B immunity? European Consensus Group on Hepatitis B Immunity. Lancet. 2000;355(9203):561-565. 
29. De Paschale M, Manco MT, Belvisi L, et al. Prevalence of markers of hepatitis B virus infection or vaccination in HBsAg-negative subjects. Blood Transfus. 2012;10(3):344-350.

30. Wu Q, Zhuang GH, Wang XL, Wang LR, Li N, Zhang M. Antibody levels and immune memory 23 years after primary plasma-derived hepatitis B vaccination: results of a randomized placebo-controlled trial cohort from China where endemicity is high. Vaccine. 2011;29(12): 2302-2307.

31. Mendy M, Peterson I, Hossin S, et al. Observational study of vaccine efficacy 24 years after the start of hepatitis B vaccination in two Gambian villages: no need for a booster dose. PLOS ONE. 2013;8(3): e58029.
32. Su FH, Bai CH, Chu FY, Lin YS, Su CT, Yeh CC. Significance and anamnestic response in isolated hepatitis $\mathrm{B}$ core antibody-positive individuals 18 years after neonatal hepatitis B virus vaccination in Taiwan. Vaccine. 2012;30(27):4034-4039.

33. Bay CE. California Association for Medical Laboratory Technology. 2012. Available from: http://www.camlt.org/pdf_files/forms/004-form. pdf.

34. Pondé RA. Atypical serological profiles in hepatitis B virus infection. Eur J Clin Microbiol Infect Dis. 2013;32(4):461-476. Available from: http://link.springer.com/article/10.1007/s10096-012-1781-9.

\section{Publish your work in this journal}

Patient Preference and Adherence is an international, peer-reviewed, open access journal focusing on the growing importance of patient preference and adherence throughout the therapeutic continuum. Patient satisfaction, acceptability, quality of life, compliance, persistence and their role in developing new therapeutic modalities and compounds to optimize clinical outcomes for existing disease states are major areas of interest. This journal has been accepted for indexing on PubMed Central. The manuscript management system is completely online and includes a very quick and fair peer-review system. Visit http://www.dovepress.com/ testimonials.php to read real quotes from published authors. 